\title{
Amateur Astronomy Network Development in Indonesia
}

\author{
Avivah Yamani ${ }^{1}$ and Hakim L. Malasan ${ }^{2}$ \\ ${ }^{1}$ langitselatan, astronomy education and communication media \\ ${ }^{2}$ Astronomy Division and Bosscha Observatory, Institut Teknologi Bandung
}

\begin{abstract}
Indonesia is a very big country with over 238 million people. And we only have one higher learning institution on astronomy, so how do we reach and convey astronomical information effectively to the whole country? The answer lies in Astronomy Clubs who play an increasingly important role to communicate and educate the public. As part of South East Asia, Indonesia is actively involved in the region to develop astronomy.
\end{abstract}

Keywords. Astronomy Club, astronomy awareness, Indonesia

\section{The role of Amateur Astronomy to build astronomy awareness}

Astronomy in Indonesia has been known since ancient times through maritime and agricultural life. Up until now, we have one Astronomy Study Program, one University Observatory, two Solar Observing Stations, three Small Observatories, four Planetaria, nineteen Astronomy Clubs, four Online Communities, and four Astronomy Media. In Indonesia, we use astronomy as an entry point to stimulate young people to learn science, culture and technology. It is also used to determine and decide the Islamic ritual dates by observing the waxing crescent. We have nineteen astronomy clubs, who introduce astronomy by conducting real astronomical activities among the public such as star parties, teacher training, etc. Astronomy Clubs also realise that collaboration with professional astronomers is very important to teach the basics of astronomy.

\section{Result and Discussion}

Challenges and problems for Clubs are the lack of instruments, resources and knowledge. Some clubs suggest having an association that conducts regular seminars. It seems that Clubs in Indonesia are not aware that we already have the Indonesia Astronomical Association (HAI) who have been conducting biannual meetings since the 1980s. To solve this problem we suggest that Indonesia needs to create a network to gather key persons from Clubs and Institutions and have suitable communication media to exchange information. Annual astronomy festivals also needed to promote astronomy and train amateurs with proper astronomical knowledge. In terms of the region, Indonesia is part of South East Asia where amateurs also play important roles to educate the public. South East Asian Astronomy Network (SEAAN) includes Public Outreach in their working groups, and South East Asian Young Astronomer Collaboration (SEAYAC) seeks amateurs as potential collaborators.

AY would like to thank LKBF and IAU for the grants to attend the $28^{\text {th }}$ IAU GA. 